\title{
Correction to: Treatment of Brucellosis in Guinea Pigs via a Combination of Engineered Novel pH-Responsive Curcumin Niosome Hydrogel and Doxycycline-Loaded Chitosan-Sodium Alginate Nanoparticles: an In Vitro and In Vivo Study
}

Fatma I. Abo El-Ela, ${ }^{1,8}$ Khaled H. Hussein, ${ }^{2}$ Hossny A. El-Banna, ${ }^{3}$ Amr Gamal, ${ }^{4}$ Sherin Rouby, Ahmed M. S. Menshawy, ${ }^{2}$ El-Shaymaa El-Nahass, ${ }^{5}$ Shehata Anwar, ${ }^{5}$ Mohamed M. A. Zeinhom, ${ }^{6}$ Heba F. Salem, ${ }^{4}$ Marawa Ahmed Yahia Al-Sayed, ${ }^{7}$ Hala A. El-Newery, ${ }^{7}$ Khaled A. M. Shokier, ${ }^{7}$

Khalid A. EL-Nesr, ${ }^{5}$ and H. I. Hosein ${ }^{2}$

published online 17 December 2020

A Correction to this paper has been published: https://doi.org/10.1208/s12249-020-01899-3

Correction to: AAPS PharmSciTech volume 21, Article number: 326 (2020) https://doi.org/10.1208/s12249-020-01833-7

Co-author EL-Shimaa EL-Nahass's name was misspelled upon publication. The correct spelling is "El-Shaymaa ElNahass".

Publisher's NoteSpringer Nature remains neutral with regard to jurisdictional claims in published maps and institutional affiliations.

The online version of the original article can be found at https:// doi.org/10.1208/s12249-020-01833-7

${ }^{1}$ Department of Pharmacology, Faculty of Veterinary Medicine, Beni-Suef University, Beni-Suef, 62511, Egypt.

${ }^{2}$ Department of Veterinary Medicine, Faculty of Veterinary Medicine, Beni-Suef University, Beni-Suef, 62511, Egypt.

${ }^{3}$ Pharmacology Department, Faculty of Veterinary Medicine, Cairo University, Cairo, Egypt.

${ }^{4}$ Department of Pharmaceutics and Industrial Pharmacy, Faculty of Pharmacy, Beni-Suef University, Beni-Suef, Egypt.

${ }^{5}$ Pathology Department, Faculty of Veterinary Medicine, Beni-Suef University, Beni-Suef, Egypt.

${ }^{6}$ Food Hygiene and Control Department, Faculty of Veterinary Medicine, Beni-Suef University, Beni-Suef, 62511, Egypt.

${ }^{7}$ Animal Health Research Institute, Beni-11Suef branch, Beni-Suef, Egypt.

${ }^{8}$ To whom correspondence should be addressed. (e-mail: fa.pharma@yahoo.com; fatma.aboel3la@vet.bsu.edu.eg) 